\title{
Treatment Patterns and Medication Use in Patients with Postherpetic Neuralgia
}

\author{
Jeffrey Gudin, MD; Jeffrey Fudin, PharmD, FCCP, FASHP; Elaine Wang, BA; \\ Todd Haylon, BA; Kalpana Patel, PharmD; and Thomas F. Goss, PharmD
}

\begin{abstract}
BACKGROUND: Postherpetic neuralgia (PHN) is a chronic, painful condition characterized by persistent pain following resolution of a herpes zoster (HZ) infection. Epidemiologic data demonstrate that the risks for $\mathrm{HZ}$ infections and the development of PHN increase with age.
\end{abstract}

OBJECTIVE: To characterize prescribing patterns, health care utilization, and treatment costs for adults with PHN based on real-world data

METHODS: This study analyzed medical and pharmacy claims from 2010 to 2014 in the MarketScan Commercial and Medicare Supplemental databases. PHN patients were identified based on criteria from a published algorithm. PHN treatment patterns were analyzed by age and reported descriptively for patients aged $<65$ or $\geq 65$ years. Excess incremental health care costs were calculated for PHN patients by comparing expenditures for a cohort of PHN patients to expenditures of a propensity score-matched control group of patients with $\mathrm{HZ}$ alone.

RESULTS: Approximately $0.4 \%$ of patients aged $<65$ years were diagnosed with $\mathrm{HZ}$ versus $1.3 \%$ of patients aged $\geq 65$ years; approximately $15.3 \%$ of $\mathrm{HZ}$ patients aged $<65$ years and $26.4 \%$ of patients aged $\geq 65$ years were diagnosed with PHN. Overall, opioids remained the most frequently prescribed initial treatment. Approximately $21.6 \%$ of PHN patients received an opioid as an initial treatment for PHN, 15.1\% received gabapentin; $8.9 \%$ received a prescription nonsteroidal anti-inflammatory drug (NSAID); $8.3 \%$ received a lidocaine patch; $3.3 \%$ received pregabalin; $2.5 \%$ received a tricyclic antidepressants (TCAs); $0.8 \%$ received other topical lidocaine; and $<1 \%$ received capsaicin. Observed first-line use of the lidocaine patch and gabapentin was higher in patients aged $\geq 65$ years relative to patients aged $<65$ years. When separated by age group, only $24.6 \%$ of patients aged $<65$ years and $38.5 \%$ of patients aged $\geq 65$ years were prescribed a recommended first-line treatment for initial PHN therapy (gabapentin, lidocaine patch, pregabalin, and TCAs). Comparisons of treatment costs of PHN patients to matched HZ patients without PHN indicated that PHN patients initiated on opioids had the highest mean additional health care expenditure compared with PHN patients initiated on other medications. On average, PHN patients initiated on opioids had $\$ 7,601$ additional health care expenditure compared with $\mathrm{HZ}$ patients with no PHN; additional expenditures were $\$ 6,428$ for pregabalin, $\$ 4,213$ for lidocaine patches, $\$ 3,478$ for gabapentin, $\$ 3,304$ for NSAIDs, and $\$ 2,797$ for TCAs, respectively.

CONCLUSIONS: Management of PHN is associated with substantial utilization of opioid-based therapies across all ages. Medications supported by evidence either as first-line therapies or as part of a multimodal regimen for the management of PHN are underused relative to opioid-based PHN therapies. Improving adherence to evidence-based PHN treatment regimens offers the potential to reduce opioid prescribing first line and reduce overall treatment costs. Given the emphasis to reduce opioid prescribing to minimize the risk of dependence, abuse, and diversion, multimodal analgesic treatments that can avoid or reduce opioid use should be considered.

J Manag Care Spec Pharm. 2019;25(12):1387-96

Copyright $\odot 2019$, Academy of Managed Care Pharmacy. All rights reserved.

\section{What is already known about this subject}

Postherpetic neuralgia (PHN) is a common consequence of herpes zoster (HZ) infection

The treatment of PHN-related pain presents a challenge for clinicians and patients, balancing efficacy and safety of available treatments. Poorly controlled PHN pain often results in multiple dosage adjustments, medication switches, and polypharmacy.

\section{What this study adds}

This study provides a contemporary assessment of the treatment patterns associated with managing PHN in a U.S. patient population of commercially insured patients, which also includes patients insured under Medicare Advantage plans; similar patterns of opioid use as a first-line PHN treatment were observed in patients aged $<65$ years and $\geq 65$ years.

This study documents the frequent use of opioids as first-line therapy in $21.6 \%$ of patients with PHN, even though this use is not supported by the current body of evidence.

This study documents the cost burden associated with PHN treatment and identifies treatment patterns associated with highest overall treatment costs, including medication switching and polypharmacy.

$\mathrm{P}$ ostherpetic neuralgia (PHN) is a complication of herpes zoster (HZ), occurring in more than 1,000,000 individuals annually in the United States. ${ }^{1-3}$ The incidence of $\mathrm{HZ}$ ranges between 2 per 1,000 in those aged 18-34 years and 10 per 1,000 in those aged $\geq 65$ years. ${ }^{4}$ While the Shingles Prevention Study (SPS) demonstrated that vaccine use reduced the HZ burden of illness by $61.1 \%$ and the incidence of PHN by $66.5 \%$, more recent Center for Disease Control surveillance reports indicate that, despite vaccination efforts, $\mathrm{HZ}$ incidence continues to rise..$^{5-7}$ Up to $34 \%$ of $\mathrm{HZ}$ patients develop PHN, characterized by pain persisting beyond 3 months after HZ infection; the incidence increases with age-patients typically are between ages 50 and 79 years—and approximately $60 \%$ of PHN cases occur in women. . $^{8-10}$

$\mathrm{HZ}$ is caused by the reactivation of varicella-zoster virus (VZV) and commonly affects the thoracic, cervical, and trigeminal nerves. ${ }^{11,12}$ Although PHN pathophysiology remains 
unclear, VZV reactivation causes a neurogenic inflammatory response that lowers the firing threshold of affected neurons generating spontaneous discharges, which along with changes in the spinal cord's signal processing, results in disproportionate pain following nonpainful stimuli (or allodynia). ${ }^{13}$ PHN pain can be burning, sharp, throbbing, stabbing, and constant or intermittent. ${ }^{14,15}$ Similar to the prodromal presentation of HZ, PHN patients frequently experience altered sensory perceptions in the affected dermatomes, such as allodynia, anesthesia, dysesthesia, and hyperalgesia. ${ }^{11,12}$ Allodynia, present in at least $70 \%$ of PHN patients, is described as the most distressing and debilitating component of PHN, as normal sensations, including clothing and light touch, become painful. ${ }^{15}$

There is no cure for PHN, and patients rely on palliative treatments to reduce pain duration and severity. ${ }^{16}$ Current evidence supports lidocaine patches, gabapentin, pregabalin, serotonin norepinephrine reuptake inhibitors, and tricyclic antidepressants (TCAs) as first-line therapies; opioid analgesics and topical capsaicin are commonly suggested as second- or third-line options. ${ }^{17-22}$

Despite the lack of evidence for use as a first-line PHN therapy, opioids are frequently prescribed first line, as documented by previous studies. ${ }^{23}$ Opioid analgesics are understood to present serious risks, including dependence, abuse, misuse, and respiratory depression, which contribute significantly to their overall cost burden. ${ }^{24,25}$ A study on opioid abuse in the United States estimated that the total health insurance costs of patients who abuse opioids exceeded $\$ 26$ billion in 2013. ${ }^{26}$ Although existing guidelines do not suggest a specific order for first-line medication use and treatment patterns may vary by patient or provider, evidence suggests that lidocaine patches and other topical nonopioid medications to treat neuropathic pain should be considered more often given their efficacy and relative lack of systemic side effects. ${ }^{23,27}$ Lidocaine patches are also considered in combination regimens, safely providing local analgesia combined with systemic therapies, especially when first-line monotherapy is partially effective. ${ }^{23}$

The U.S. Census Bureau projects continued growth in the population aged $\geq 65$ years, which, when combined with the lack of effective treatment options, confirms that the burden of PHN in the United States will likely remain high, from a patient quality of life and cost perspective. ${ }^{6}$ This is despite the introduction of the newest adjuvanted $\mathrm{HZ}$ subunit ( $\mathrm{HZ} / \mathrm{su}$ ) vaccine (Shingrix, GSK). ${ }^{5}$ Although the 6-year postvaccination data for the HZ/su shingles vaccine demonstrated that the product has an estimated efficacy of 50\%-99\% for preventing HZ (and therefore PHN), long-term effectiveness may be limited based on analysis of cellular and humoral response after 6 years postvaccination with the conjugated vaccine. ${ }^{28}$

Patients with PHN have been reported to incur expenditures 2-4 times higher than patients with $\mathrm{HZ}$ alone. ${ }^{29}$ The majority of additional expenditures observed in PHN patients were attributed to nonemergency outpatient (50\%) and pharmacy (25\%) claims. $^{29}$ Moreover, PHN patients were reported to have significantly higher rates of interventional treatments or procedures (spinal injections, nerve blocks, stimulator implantation, transcutaneous nerve stimulation, and trigger point injections). ${ }^{30}$

The primary objective of this study was to identify treatment patterns in PHN management. The secondary objective was to characterize health care utilization and expenditure associated with PHN diagnosis and treatment.

\section{Methods}

\section{Study Design and Data Source}

This study was a retrospective cohort analysis using claims data to assess treatment patterns, health care utilization, and expenditures in PHN patients diagnosed from 2010 to 2014.

Deidentified patient-level data were obtained from Truven Health MarketScan (now IBM MarketScan) Commercial Claims and Encounters and Medicare Supplemental and Coordination of Benefits databases. These databases include large, nationally representative data documenting the health services of approximately 232 million employees, dependents, and retirees in the United States with primary or Medicare supplemental coverage through privately insured fee-for-service, point-of-service, or capitated health plans. Enrollment records, inpatient, outpatient, ancillary, and drug claims from 2010 to 2014 were analyzed for this study. This study did not meet the federal definition of "human subject research" and was exempt from review by the institutional review board.

\section{Patient and Cohort Identification}

PHN Patients. Unlike HZ, PHN did not have a unique International Classification of Diseases, Ninth Revision, Clinical Modification (ICD-9-CM) code in use at the time this analysis was conducted. As such, clinicians did not consistently code PHN, and the true incidence of PHN was underestimated in retrospective analyses. To address this, PHN patients were identified in our study using an optimized and validated algorithm reported by Klompas et al. (2011) incorporating the following 3 criteria that increased detection of $\mathrm{HZ}$ cases progressing to PHN from 5.4\% to $17.8 \%{ }^{31}$ : (1) had an ICD-9-CM code for HZ with nervous system complications 053.1X); (2) had an ICD-9-CM code for neuralgia (792.2) within 180 days of a visit for HZ (053.X); or (3) had two 053.X ICD-9-CM visits (HZ) within 21 days plus a prescription for an analgesic, anticonvulsant, or antidepressant within 60 days (prescriptions were aggregated by Anatomical Therapeutic Chemical class, and individual prescription medications were identified by National Drug Code number).

PHN patients were identified by a diagnosis of $\mathrm{HZ}$ and having (a) met PHN diagnosis criteria reported by Klompas et al. ${ }^{31}$ or (b) received newly prescribed treatment with a lidocaine patch within 60 days of $\mathrm{HZ}$ diagnosis. Because the only U.S 


\section{FIGURE 1 Methodology for Creation of Study Cohort ${ }^{31}$}
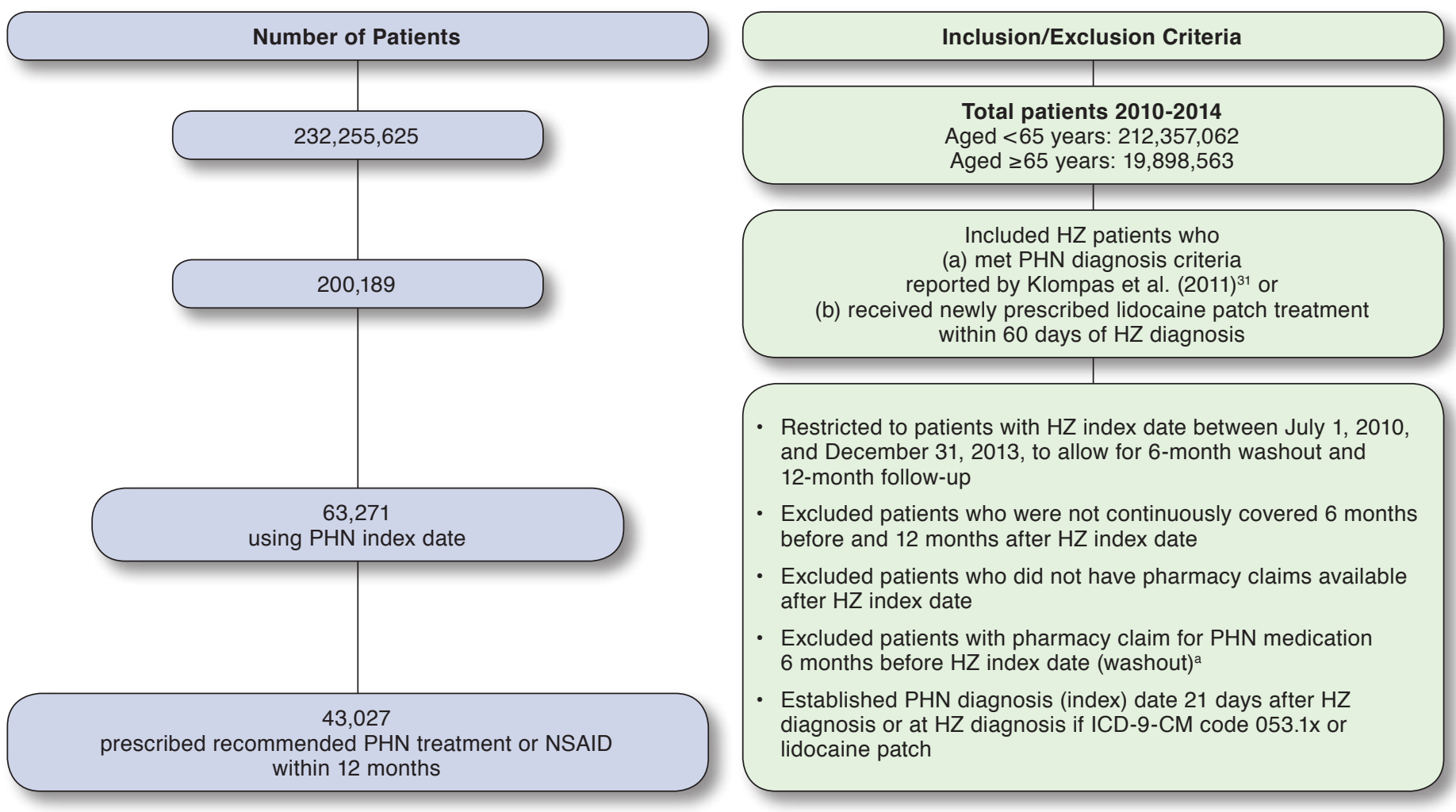

aLidocaine patch, topical lidocaine, gabapentin, pregabalin, tricyclic antidepressant, opiate agonist/partial agonist, capsaicin, or prescription NSAID.

HZ=herpes zoster; ICD-9-CM=International Classification of Diseases, Ninth Revision, Clinical Modification; NSAID=nonsteroidal anti-inflammatory drug; PHN=postherpetic neuralgia.

Food and Drug Administration (FDA)-approved indication for the lidocaine patch is PHN, when it was prescribed after a HZ diagnosis, the research team accepted this use as likely prescribed for PHN.

HZ Alone Control Patients. Controls were selected per the method used in Dworkin et al. (2011). ${ }^{32}$ Subjects had a diagnosis of HZ, but no diagnosis of PHN. Control subjects were selected using the following ICD-9-CM codes: 053.0, 053.10, 053.11, 053.14, 053.2x, 053.8, or 053.9.

PHN Cohort. To allow for a 6-month washout period and a 12-month follow-up period, the study cohort was restricted to PHN patients who were diagnosed with HZ between July 1, 2010, and December 31, 2013 (HZ index date). Additional exclusion criteria included (a) patients who were not continuously covered by insurance 6 months before and 12 months after HZ index date; (b) patients who did not have pharmacy claims available after the HZ index date; and (c) patients with a pharmacy claim for PHN medication (lidocaine patch, topical lidocaine, gabapentin, pregabalin, tricyclic antidepressant, opiate agonist/partial agonist, capsaicin, or prescription NSAID) 6 months before $\mathrm{HZ}$ index date.

A flow chart of the selection process is shown in Figure 1.

\section{Assessment of Treatment Patterns, Health Care Utilization, and Expenditure}

Treatment patterns were based on observation of newly initiated medications in the 12-month period following a PHN diagnosis. Current evidence suggests gabapentin, pregabalin, lidocaine patch, or TCAs be considered as the first-line PHN treatment options, with either opioids or capsaicin as a second- or third-line therapy, and do not support NSAIDs as a PHN treatment. ${ }^{16-22}$ The addition of a second medication to the initial medication ("add") was defined as a pharmacy claim for a second PHN medication at least 30 days before completion of the initial medication, combined with refill of the initial medication. Switching from the initial medication to a second medication ("switch") was defined as a pharmacy claim for a second PHN medication fewer than 30 days before the completion date of the initial medication. For the purposes of this analysis, 


\section{FIGURE 2 Initial Medication Use Among PHN} Patients $(n=63,271)^{a}$

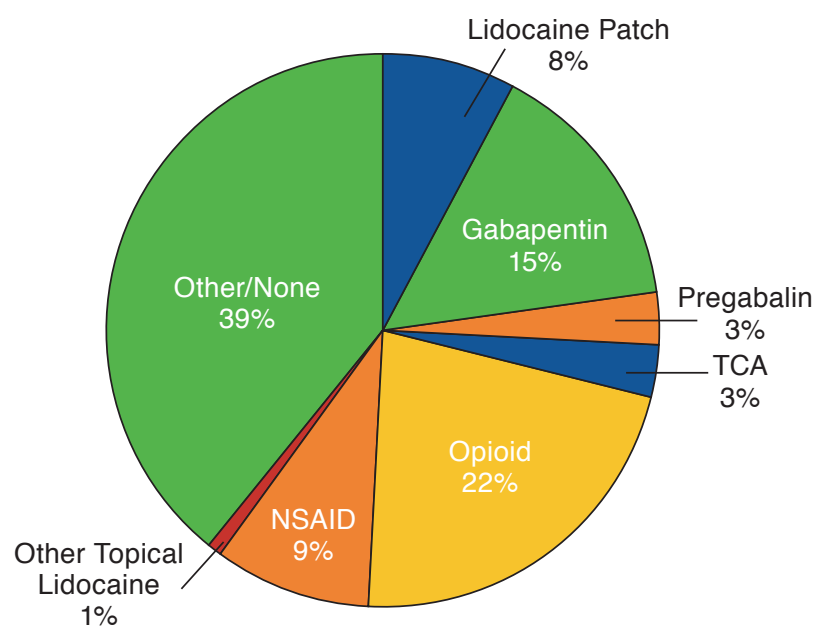

a Because capsaicin was $<1 \%$, it is not displayed.

NSAID = nonsteroidal anti-inflammatory drug; $P H N=$ postherpetic neuralgia; $T C A=$ tricyclic antidepressant.

topical lidocaine not in patch formulation was not considered first line, as the International Association for the Study of Pain (IASP) specifically recommends the patch formulation. ${ }^{33}$

\section{Statistical Analyses}

Descriptive statistical analyses of the study cohort summarized mean and standard deviation (SD) for continuous variables and frequencies and proportions for categorical variables.

The treatment patterns and health care expenditure cost estimates were provided for all patients and stratified by patients aged $<65$ and $\geq 65$ years. To assess the PHN treatment patterns of patients newly initiated on medications following PHN diagnosis, descriptive statistics were used to summarize the prescribed PHN treatments during the 12-month follow-up period. Although NSAID therapy is not recommended for treatment of PHN, given the temporal relationship between PHN diagnosis and new NSAID prescriptions, we have reported NSAIDS in this descriptive analysis. Comparisons of continuous variables were performed using t-tests and categorical variables using Pearson chi-square tests. Analyses were performed in SAS software version 9.4 (SAS Institute, Cary, NC), and 2-sided comparisons were performed at a $P=0.05$ level of significance.

\section{Results}

\section{Study Population}

Of the total patients identified from the databases from 2010 to $2014,0.4 \%$ of the patients aged $<65$ years and $1.3 \%$ aged $\geq 65$ years were diagnosed with HZ. Of those aged $<65$ and $\geq 65$ years diagnosed with $\mathrm{HZ}, 15.2 \%$ and $26.4 \%$ of patients, respectively, were subsequently diagnosed with PHN, and patterns of initial analgesic use were reviewed.

Based on newly initiated treatments after diagnosis, treatment patterns were reported for 63,271 patients, of whom 42,465 were aged $<65$ years and 20,806 were aged $\geq 65$ years. Of the 63,271 patients, 43,027 patients were prescribed a recommended PHN treatment or NSAID within 12 months. Based on this observation, approximately $32.0 \%$ of PHN patients identified did not need prescription medication or possibly did not have PHN, which appears to be in line with the approximately $78 \%$ positive predictive value reported by Klompas et al. ${ }^{31}$ To assess the incremental health care utilization and expenditures incurred by each PHN patient relative to an $\mathrm{HZ}$ alone control patient, 33,975 matched PHN patients were identified.

\section{Treatment Patterns and Adherence to Guidelines}

The first-line use of opioids in the treatment of neuropathic pain (including PHN) remains controversial. The American Academy of Neurology released guidelines for management of PHN in 2004 and included controlled-release oxycodone or morphine sulfate among first-line treatment recommendations; the guidelines were subsequently retired in 2018. ${ }^{34}$ In contrast, in a 2012 study, Dworkin et al. similarly used published evidence-based recommendations from the IASP Neuropathic Pain Special Interest Group (NeuPSIG) in 2007, in which they acknowledged this lack of consensus and further stated, "Neither the more recent NeuPSIG recommendations nor any other current recommendations consider opioids and tramadol first-line treatments." ${ }^{23}$ For this analysis, the recommendations published in Dworkin et al. were in place throughout the time of the analysis period and were used as a reference against which to assess treatment patterns, health care utilization and expenditures. The current available evidence continues to support these recommendations. ${ }^{16-22}$

Only 29.2\% of all patients' initial treatment was recommended as a first-line PHN treatment, including 15.1\% gabapentin, $8.3 \%$ lidocaine patch, 3.3\% pregabalin, and $2.5 \%$ TCAs.

Nearly $22 \%$ of patients' initial treatments were recommended as second or third line, with virtually all of those patients being prescribed opioids $(<1 \%$ were prescribed capsaicin), ${ }^{16-22}$ and $9 \%$ of patients were prescribed topical or oral prescription NSAIDs as initial treatment, which are not recommended by existing evidence and are not thought to be effective in treating neuropathic pain by consensus guidelines. The remainder of PHN patients received either other or no medication for their initial PHN therapy (39\%; Figure 2).

Of note, opioids were the most commonly prescribed initial treatment (22\% of patients), despite being a second- or thirdline treatment in guideline recommendations. Gabapentin, a first-line treatment recommendation, was the second most 


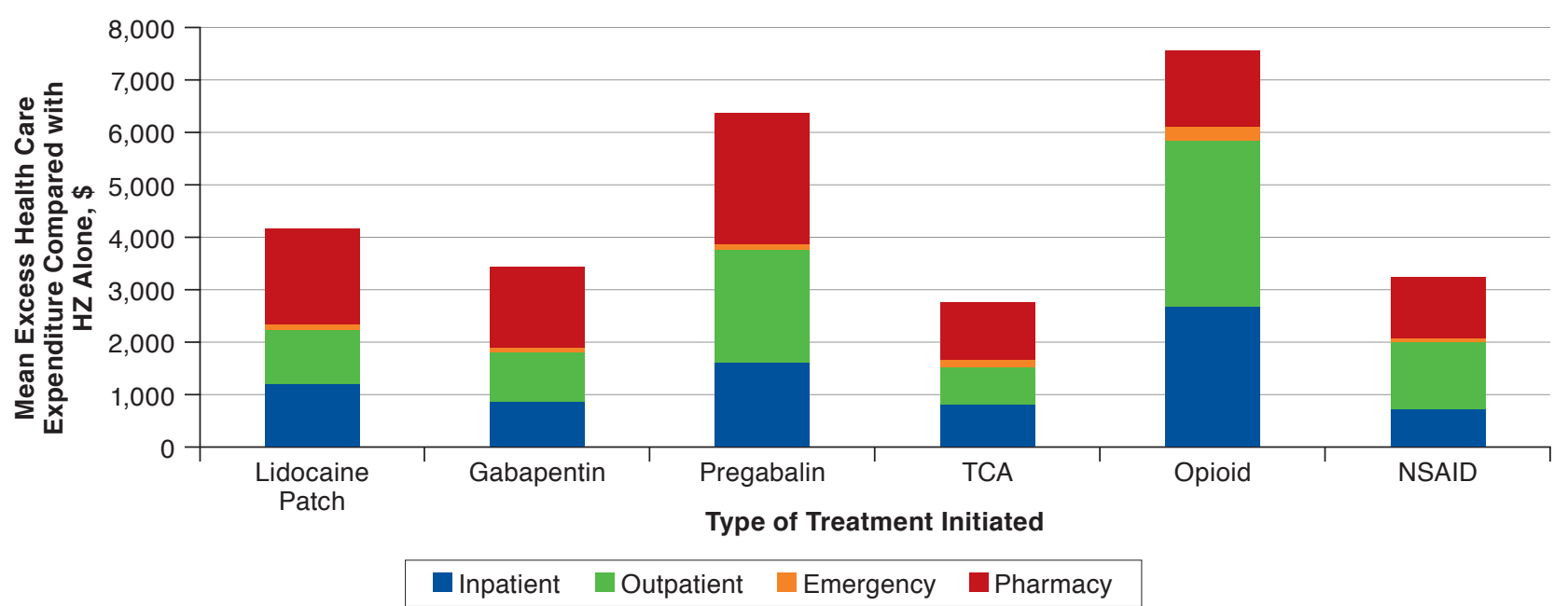

${ }^{a}$ Capsaicin and other topical lidocaine are not shown because their excess health care expenditures were not statistically significant relative to $\mathrm{HZ}$ alone. $H Z=$ herpes zoster; NSAID = nonsteroidal anti-inflammatory drug; $P H N=$ postherpetic neuralgia; TCA = tricyclic antidepressant.

frequently prescribed first-line treatment, used in 15\% of patients.

When evaluated by age group, $25 \%$ of patients aged $<65$ years and $39 \%$ of patients aged $\geq 65$ years were prescribed a first-line recommended treatment for initial PHN therapy (gabapentin, lidocaine patch, pregabalin, and TCAs). Opioids remained the most frequently prescribed initial treatment in PHN patients (21\% of patients aged $<65$ years and $23 \%$ of patients aged $\geq 65$ years were prescribed opioids as an initial therapy). Gabapentin was the second most frequently initiated medication in both age groups ( $12 \%$ in age $<65$ years and $21 \%$ in age $\geq 65$ years). Lidocaine patches were the third most frequently initiated medication in patients aged $\geq 65$ years (10\%), but the fourth most frequently initiated medication in patients aged $<65$ years $(7 \%)$. NSAIDs were the third most frequently initiated medication in patients aged $<65$ years $(11 \%)$, but the fourth most frequently initiated medication in patients aged $\geq 65$ years (6\%). Of note, NSAIDs were more frequently used before the recommended first- or second-line treatments in patients aged $<65$ years compared with patients aged $\geq 65$ years. In addition, more than $54 \%$ of patients aged $<65$ years and $39 \%$ of patients aged $\geq 65$ years received either a nonrecommended medication, no medication, or other medication for initial PHN treatment.

\section{Health Care Utilization and Expenditure}

PHN patients initiated on opioids for PHN treatment had the highest mean additional health care expenditure relative to patients initiated on other medications. On average, PHN patients initiated on opioids had $\$ 7,601(P<0.05)$ excess additional health care expenditure compared with $\$ 6,428(P<0.05)$ for pregabalin, $\$ 4,213(P<0.05)$ for lidocaine patches, $\$ 3,478$ $(P<0.05)$ for gabapentin, $\$ 3,304(P<0.05)$ for NSAIDs, and $\$ 2,797(P<0.05)$ for TCAs. The majority of additional mean health care costs in patients of all ages prescribed opioids were attributed to inpatient and outpatient visits, $\$ 2,677$ and $\$ 3,190$ respectively (Figure 3).

When evaluated by age group, patients aged $<65$ and $\geq 65$ years who were initiated on opioids had the highest mean additional health care expenditure relative to patients initiated on other medications. PHN patients aged $<65$ years initiated on opioids had a mean additional health care expenditure of $\$ 7,929$, and patients aged $\geq 65$ years initiated on opioids had a mean additional health care expenditure of $\$ 6,943$. Among patients initiated on opioids, outpatient visit claims constituted the highest proportion of the mean additional health care expenditure for those aged $<65$ years; whereas, for those aged $\geq 65$ years, inpatient visit claims constituted the highest proportion of additional health care costs. On average, the second highest additional health care expenditure for those aged $<65$ years were PHN patients initiated on pregabalin $(\$ 7,871)$ and for those aged $\geq 65$ years were PHN patients initiated on lidocaine patches $(\$ 5,335)$.

Among patients who switched or added a second medication, a higher proportion of PHN patients switched to a second medication, with $18 \%-36 \%$ of patients switching, compared with patients who added a second medication as noted in Figure 4. Among patients who added or switched to a 


\section{FIGURE 4 Percentage of PHN Patients Initiated on a Guideline-Recommended Treatment Who Added a Second} Medication, Switched to a Second Medication, or Neither, Sorted by First Medication Initiated

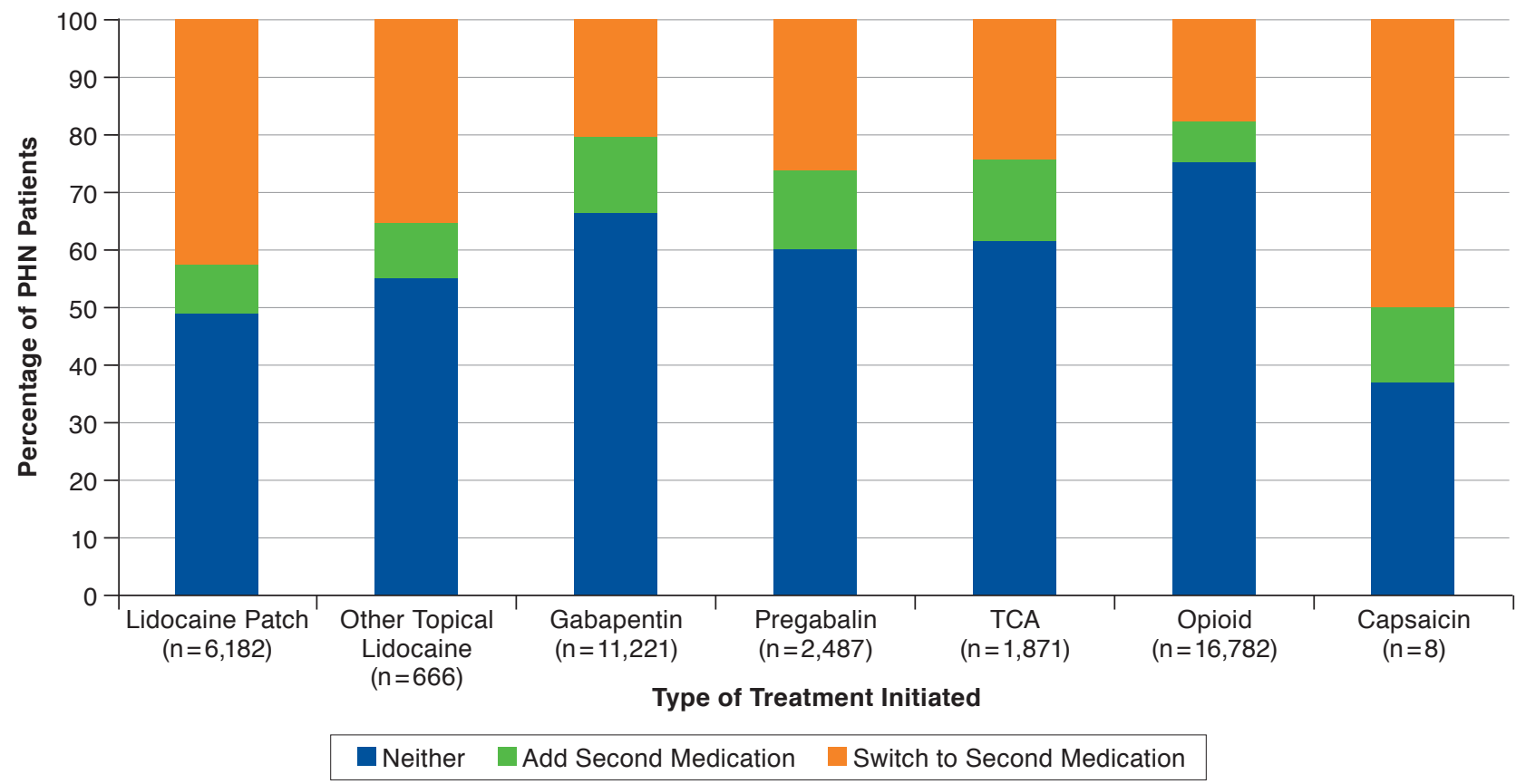

PHN= postherpetic neuralgia; TCA = tricyclic antidepressant

second medication, opioids were the most frequently prescribed second medication. Of PHN patients initiated on a lidocaine patch, $49 \%$ stayed on the lidocaine patch as a first-line therapy; $43 \%$ switched to another medication; and $8 \%$ added a second medication to the lidocaine patch (Figure 4).

Adding or switching an initial PHN medication was associated with higher mean additional health care expenditure when compared with PHN patients who did not add or switch their initial medication. The mean additional health care expenditures associated with adding a second medication were highest for those initiated on lidocaine patches, and the additional expenditure for switching a medication were highest for those initiated on TCAs and capsaicin. Of patients who were initially started on lidocaine patches and switched to a second medication, opioids were the most frequently prescribed, with $53 \%$ of the patients who switched moving to opioids. Of the patients who were initially prescribed a lidocaine patch and added a second medication, opioids were the most frequent addition, with $40 \%$ of patients adding a medication adding an opioid to initial treatment. Adding an opioid is associated with the highest mean additional total health care cost of $\$ 11,522$ relative to other second medications added: $\$ 11,408$ for pregabalin, $\$ 10,296$ for NSAIDs, $\$ 8,575$ for TCAs, and $\$ 8,225$ for gabapentin.
PHN patients initiated on gabapentin, pregabalin, or TCA who did not add or switch to a second medication had similar health care expenditure to matched $\mathrm{HZ}$ controls $(P \geq 0.05)$; all other groups were statistically significantly different from matched HZ controls $(P<0.05$; Figure 5$)$.

\section{Discussion}

The substantial clinical and economic burden associated with PHN demonstrates the need for more cost-efficient treatment practices. PHN may be refractory to treatment, and most first-line medications have common systemic side effects, (sedation, dry mouth, blurred vision, weight gain, urinary retention, peripheral edema, and others). ${ }^{29}$ Managing side effects is associated with longer titration, additional physician visits, abandoned medications, switching, and polypharmacy, which increase treatment costs. $915,29,30,32,35$ Furthermore, PHN pain substantially lowers health-related quality-of-life scores (reported health utility of 0.61 for PHN patients compared with 0.87 for the general population norm), severely affects patients' abilities to carry out general activities, and is associated with significant work productivity losses..$^{36-39}$

We note that prevalence estimates for $\mathrm{HZ}$ by age in this cohort are not directly comparable to other published estimates such as Leung et al. (2011) for several possible reasons. ${ }^{4}$ The 
FIGURE 5 Mean Excess Health Care Expenditure Among PHN Patients Who Added a Second Medication, Switched to a Second Medication, or Neither, Sorted by First Medication Initiated ${ }^{a}$

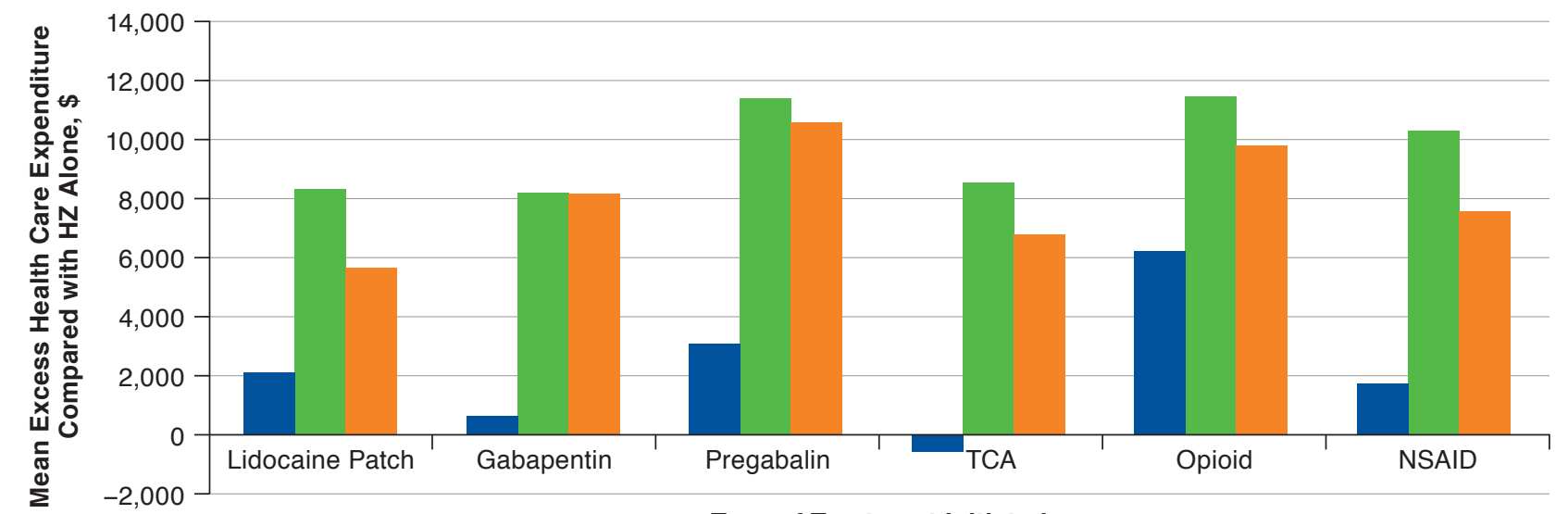

Type of Treatment Initiated

Neither Add Second Medication $\quad$ Switch to Second Medication

aPatients who were initiated on gabapentin, pregabalin, or TCA and did not add or switch to a second medication did not have excess health care expenditures that differed significantly from matched $\mathrm{HZ}$ controls $(P \geq 0.05)$; all other groups were statistically significantly different from matched $\mathrm{HZ}$ controls $(P<0.05)$.

NSAID = nonsteroidal anti-inflammatory drug; $P H N=$ postherpetic neuralgia; TCA = tricyclic antidepressant.

respective cohorts cover different periods of time when $\mathrm{HZ}$ vaccination rates were changing, which may affect comparisons. Also, we excluded potential cases in our cohort if they did not remain continuously eligible throughout our entire study period, whereas Leung et al. estimated annual rates. ${ }^{4}$

The results of this study suggest that there is underutilization of evidence-based recommended first-line treatments for PHN. Medications that are supported for second- or third-line treatment (e.g., opioids) or other medications that are not supported by evidence (e.g., NSAIDs) are frequently used as initial therapy for PHN. As with any complex pain pathology, the most effective approach appears to be employment of multimodal analgesic therapies with synergistic effects. ${ }^{17,40-42}$ Current evidence supports gabapentin, pregabalin, lidocaine patch 5\%, and TCAs as first-line treatment options; opioid analgesics and topical capsaicin are commonly suggested as second- or third-line therapeutic options. ${ }^{16-22,24}$ However, our data document that evidence-based PHN treatment regimens are not routinely followed, suggesting opportunities to improve patient care through more consistent adherence with current specialty society treatment recommendations. ${ }^{36}$

PHN patients started on opioids have higher incremental mean health care costs relative to matched HZ control patients than those patients started on other medications: $\$ 7,601$ for opioids relative to $\$ 6,428$ for pregabalin, $\$ 4,213$ for lidocaine patches, $\$ 3,478$ for gabapentin, $\$ 3,304$ for NSAIDs, and $\$ 2,797$ for TCAs. The majority of additional costs related to opioids are associated with inpatient and outpatient visits, which results in $\$ 2,677$ and $\$ 3,190$ additional health care costs, respectively. Additionally, NSAIDs generate substantial additional costs $(\$ 3,304)$ and inherent adverse effects despite not being supported by evidence for the treatment of PHN.

We observed that a higher percentage of patients switch from first-line lidocaine patches to another medication compared with those started on any other first-line PHN medication. Only 49\% of PHN patients who initiate a lidocaine patch as first-line therapy stay on the lidocaine patch alone. The remaining $43 \%$ of PHN patients who initiate a lidocaine patch as first-line therapy switch to another medication, and $8 \%$ add a second medication. Of patients started on a lidocaine patch who switch to a second medication, the most common switch made is to opioids (53\%). Of patients who started on lidocaine patch who add a second medication, $40 \%$ add an opioid. Adding opioids as a second medication is associated with the highest mean excess total health care costs relative to other second medications: $\$ 11,522$ for adding opioids compared with $\$ 11,408$ for pregabalin, $\$ 10,296$ for NSAIDs, $\$ 8,575$ for TCAs, and $\$ 8,225$ for gabapentin.

Reasons for frequent switching from lidocaine patches to another therapy are thought to be multifactorial and may suggest that in some patients either the condition itself is refractory to treatment with lidocaine patches or there may be shortcomings in the patch formulations that were available at the time of this analysis; approximately $70 \%$ of the total cases 
reported to the FDA Adverse Events Reporting System for the lidocaine patch are related to product adhesion, a much higher rate than for other patch products, suggesting that patch adhesion may be 1 reason patients switch away from lidocaine patch therapy. ${ }^{43,44}$ These adhesion issues are important as current evidence suggests that sustained adhesion of a patch to the skin improves the delivery of the drug from the patch. ${ }^{45,46}$

\section{Limitations}

There are several potential limitations in this study. Some limitations are inherent to the nature of claims analysis studies, including potential coding errors. Because a specific ICD-9-CM code for PHN was unavailable at the time of analysis, it is possible that there was misclassification of those who actually had PHN and those who had HZ. However, we attempted to address this potential limitation by using a validated method of identification developed by Klompas et al. ${ }^{31}$

As previously noted, a potential limitation may have resulted from the modification of the Klompas et al. definition to include patients who had an HZ diagnosis accompanied by a claim for a lidocaine patch. The basis for this modification is that the only FDA-approved label indication for the lidocaine patch is PHN, suggesting that the combination of an $\mathrm{HZ}$ diagnosis and lidocaine patch is a valid indicator of PHN. We recognize that despite the PHN indication, lidocaine patches may be prescribed for other conditions and that the effect of this assumption on our findings is unknown.

Because more specific International Classification of Diseases, Tenth Revision, Clinical Modification (ICD-10-CM) codes are now available, an updated analysis using ICD-10-CM diagnosis codes could be informative. We were also unable to determine symptom severity from diagnosis codes and therefore cannot differentiate our findings based on symptom severity. We did not perform an analysis comparing total duration of treatment, which in retrospect may have been useful.

Another potential limitation in our study is that the incidence of PHN may vary by geography and provider organization. The databases we used for our analyses have greater representation from certain regions of the United States, which could have resulted in skewed health care utilization and cost findings that we did not adjust for.

We also note that the generalizability of the Klompas et al. algorithm, which was initially validated in 1 health system with limited geographic diversity, to a national sample has not been validated. It is possible that specific patterns of coding, diagnostic, and medication prescribing (based on formulary limitations) are not entirely generalizable. We do note, however, that in spite of this limitation, Klompas et al. suggest in their publication that if their algorithm is affirmed, it can be used to obtain national estimates of PHN incidence in Medicare data. ${ }^{31}$ Although the algorithm may be imperfect, we believe it improved case ascertainment, given the lack of a unique ICD-9-CM code for PHN at the time of this study.
Finally, a limitation of retrospective observational studies is that confounding factors may bias results because of the lack of randomization in assigning individuals to groups. To control for variables that may potentially introduce bias, propensity score matching was used to control for demographic characteristics and comorbidities.

\section{Conclusions}

The results of this study indicate that there is underuse of evidence-based recommended first-line treatments for PHN. Our findings also demonstrate that substantial departure from evidence-based treatment recommendations for first-line therapy for PHN, suggesting that there is an unmet need for effective therapies that provide pain relief while minimizing systemic side effects. Medications that are supported for second- or third-line treatment (e.g., opioids) or other medications that are not supported by evidence (e.g., NSAIDs) are frequently prescribed as initial therapy for PHN patients. This analysis also demonstrates that there are high additional costs associated with using opioids as an initial or add-on PHN treatment. Additionally, the frequent switching and/or adding of medications also drives significant additional costs among PHN patients.

\section{Authors}

JEFFREY GUDIN, MD, Rutgers New Jersey Medical School, Newark. JEFFREY FUDIN, PharmD, FCCP, FASHP, Remitigate, Delmar, New York, and Albany College of Pharmacy \& Health Sciences, Albany, New York. ELAINE WANG, BA; TODD HAYLON, BA; and THOMAS F. GOSS, PharmD, Boston Healthcare Associates, Boston, Massachusetts. KALPANA PATEL, PharmD, SCILEX Pharmaceuticals, Mission Viejo, California.

AUTHOR CORRESPONDENCE: Thomas F. Goss, PharmD, Boston Healthcare Associates, 33 Arch St., 17th Fl., Boston, MA 02110. Tel.: 617-912-6590; E-mail: Tgoss@bostonhealthcare.com.

\section{DISCLOSURES}

Research funding was provided by SCILEX Pharmaceuticals. The sponsor reviewed and approved the research plan and provided support for manuscript preparation through Patel's role as a coauthor of this manuscript. The sponsor's product (lidocaine patch) was not used in this study.

Patel is a paid employee of SCILEX Pharmaceuticals. Goss is an employee and minority owner of Boston Healthcare Associates, which received a research grant from SCILEX Pharmaceuticals to conduct this study. Gudin reports advisory board fees from AcelRx Pharmaceuticals and BioDelivery Sciences International and consulting fees from Averitas, Daiichi, Hisumitsu, Nektar, Purdue, Quest Diagnostics, SCILEX Pharmaceuticals and US WorldMeds, unrelated to this study. Fudin reports advisory board fees from AcelRx Pharmaceuticals, Human Half-Cell, Quest Diagnostics, GlaxoSmithKline, SCILEX Pharmaceuticals, BioDelivery Sciences, Daiichi Sankyo, and Salix Pharmaceuticals; speaker fees from Daiichi Sankyo, Salix Pharmaceuticals, Abbott Laboratories, Acutis Diagnostics, and AstraZeneca; and consulting fees from Firstox Laboratories, unrelated to this study. The other authors have nothing to disclose. 
Parts of this research were presented at the AMCP Managed Care \& Specialty Pharmacy Annual Meeting; April 22, 2016; San Francisco, CA, and at the 35th Annual Scientific Meeting of the American Pain Society; May 11-14, 2016; Austin, TX.

\section{ACKNOWLEDGMENTS}

The research team would like to acknowledge the thoughtful contributions of Jennifer Gilbert, PhD.

\section{REFERENCES}

1. Suaya JA, Chen SY, Li Q, Burstin SJ, Levin MJ. Incidence of herpes zoster and persistent post-zoster pain in adults with or without diabetes in the United States. Open Forum Infect Dis. 2014;1(2):ofu049.

2. Glausner TA, Salinas GD, Nevins H, Williamson JC, Wallace MS, Abdolrasulnia M. Communication gaps between physicians and patients with postherpetic neuralgia: results from a national study on practice patterns. J Pain Res. 2011;4:407-15.

3. Adams EN, Parnapy S, Bautista P. Herpes zoster and vaccination: a clinical review. Am J Health Syst Pharm. 2010;67(9):724-27.

4. Leung J, Harpaz R, Molinari NA, Jumaan A, Zhou F. Herpes zoster incidence among insured persons in the United States, 1993-2006: evaluation of the impact of varicella vaccination. Clin Infect Dis. 2011;52(3):332-40.

5. Oxman MN, Levin MJ, Johnson GR, et al; Shingles Prevention Study Group. A vaccine to prevent herpes zoster and postherpetic neuralgia in older adults. N Engl J Med. 2005;352(22):2271-84.

6. Center for Disease Control and Prevention. Shingles (herpes zoster). Last reviewed August 14, 2019. Available at: https://www.cdc.gov/shingles/surveillance.html. Accessed September 6, 2019.

7. Johnson BH, Palmer L, Gatwood J, Lenhart G, Kawai K, Acosta CJ. Annual incidence rates of herpes zoster among an immunocompetent population in the United States. BMC Infect Dis. 2015;15:502.

8. Johnson P, Becker L, Halpern R, Sweeney M. Real-world treatment of post-herpetic neuralgia with gabapentin or pregabalin. Clin Drug Investig. 2013;33(1):35-44.

9. Kirson NY, Ivanova JI, Birnbaum HG, et al. Comparing healthcare costs of Medicaid patients with postherpetic neuralgia (PHN) treated with lidocaine patch 5\% versus gabapentin or pregabalin. J Med Econ. 2010;13(3):482-91.

10. Fashner J, Bell AL. Herpes zoster and postherpetic neuralgia: prevention and management. Am Fam Physician. 2011;83(12):1432-37.

11. Bowsher D. Pathophysiology of postherpetic neuralgia: towards a rational treatment. Neurology. 1995;45(12 Suppl 8):S56-57.

12. Weaver BA. The burden of herpes zoster and postherpetic neuralgia in the United States. J Am Osteopath Assoc. 2007;107(3 Suppl 1):S2-S7.

13. Kost RG, Straus SE. Postherpetic neuralgia: pathogenesis, treatment, and prevention. N Engl J Med. 1996;335(1):32-42.

14. Dworkin RH, Portenoy RK. Pain and its persistence in herpes zoster. Pain. 1996;67(2-3):241-51.

15. Johnson RW, Wasner G, Saddier P, Baron R. Herpes zoster and postherpetic neuralgia optimizing management in the elderly patient. Drugs Aging. 2008;25(12):991-1006

16. Massengill JS, Kittredge JL. Practical considerations in the pharmacological treatment of postherpetic neuralgia for the primary care provider. J Pain Res. 2014;7:125-32

17. Argoff CE. Review of current guidelines on the care of postherpetic neuralgia. Postgrad Med. 2011;123(5):134-42.

18. Attal N, Cruccu G, Baron R, et al. EFNS guidelines on the pharmacological treatment of neuropathic pain: 2010 revision. Eur J Neurol. 2010;17(9):1113-e88
19. Finnerup NB, Attal N, Haroutounian S, et al. Pharmacotherapy for neuropathic pain in adults: systematic review and meta-analysis. Lancet Neurol. 2015;14(2):162-73.

20. Hooten WM, Timming R, Belgrade M, et al.; Institute for Clinical Systems Improvement. Health care guideline: assessment and management of chronic pain. 2013. Available at: http://formsus.datasus.gov.br/novoimgarq/33266/6201476_353278.pdf. Accessed September 5, 2019

21. Moulin D, Boulanger A, Clark AJ, et al. Pharmacological management of chronic neuropathic pain: revised consensus statement from the Canadian Pain Society. Pain Res Manag. 2014;19(6):328-35.

22. Stankus SJ, Dlugopolski M, Packer D. Management of herpes zoster (shingles) and postherpetic neuralgia. Am Fam Physician. 2000;61(8):2437-44

23. Dworkin RH, Panarites CJ, Armstrong EP, Malone DC, Pham SV. Is treatment of postherpetic neuralgia in the community consistent with evidence-based recommendations? Pain. 2012;153(4):869-75.

24. Dowell D, Haegerich TM, Chou R. CDC guideline for prescribing opioids for chronic pain-United States, 2016. MMWR Recomm Reports. 2016;65(1):1-49.

25. Birnbaum HG, White AG, Schiller M, Waldman T, Cleveland JM, Roland CL. Societal costs of prescription opioid abuse, dependence, and misuse in the United States. Pain Med. 2011;12(4):657-67.

26. Florence CS, Zhou C, Luo F, Xu L. The economic burden of prescription opioid overdose, abuse, and dependence in the United States, 2013. Med Care. 2016;54(10):901-06.

27. Dworkin RH, O'Connor AB, Backonja M, et al. Pharmacologic management of neuropathic pain: evidence-based recommendations. Pain. 2007;132(3):237-51

28. Chlibek R, Pauksens K, Rombo L, et al. Long-term immunogenicity and safety of an investigational herpes zoster subunit vaccine in older adults. Vaccine. 2016;34(6):863-68.

29. Dworkin RH, White R, O'Connor AB, Baser O, Hawkins K. Healthcare costs of acute and chronic pain associated with a diagnosis of herpes zoster. J Am Geriatr Soc. 2007;55(8):1168-75.

30. Dworkin RH, White R, O'Connor AB, Baser O, Hawkins K. Health care expenditure burden of persisting herpes zoster pain. Pain Med. 2008;9(3):348-53

31. Klompas M, Kulldorff M, Vilk Y, Bialek S, Harpaz R. Herpes zoster and postherpetic neuralgia surveillance using structured electronic data. Mayo Clin Proc. 2011;86(12):1146-53.

32. Dworkin RH, Panarites CJ, Armstrong EP, Malone DC, Pham SV. Healthcare utilization in people with postherpetic neuralgia and painful diabetic peripheral neuropathy. J Am Geriatr Soc. 2011;59(5):827-36.

33. Dworkin RH, Robert H, Backonja M, et al. Pharmacologic management of neuropathic pain: evidence-based recommendations. Pain. 2007;132(3):237-51.

34. Dubinsky RM, Kabbani H, El-Chami Z, Boutwell C, Ali H. Practice parameter: treatment of postherpetic neuralgia: an evidence-based report of the Quality Standards Subcommittee of the American Academy of Neurology. Neurology. 2004;63(6):959-65.

35. Gudin J, Backonja M, Nalamachu S. A review of the clinical data on ZTildo (lidocaine patch 1.8\%). Postgraduate Med. 2018;130(Suppl 1):S45.

36. Doth AH, Hansson PT, Jensen MP, Taylor RS. The burden of neuropathic pain: a systematic review and meta-analysis of health utilities. Pain. 2010;149(2):338-44.

37. Gater A, Uhart M, McCool R, Preaud E. The humanistic, economic and societal burden of herpes zoster in Europe: a critical review. BMC Public Health. 2015;15:193.

38. Oster G, Berger A, Dukes E, Edelsberg J, McCarberg B. Use of potentially inappropriate pain-related medications in older adults with painful neuropathic disorders. Am J Geriatr Pharmacother. 2004;2(3):163-70. 
39. Lamers LM, Meerding WJ, Severens JL, Brouwer WB. The relationship between productivity and health-related quality of life: an empirical exploration in persons with low back pain. Qual Life Res. 2005;14(3):805-13.

40. White WT, Patel N, Drass M, et al. Lidocaine patch 5\% with systemic analgesics such as gabapentin: a rational polypharmacy approach for the treatment of chronic pain. Pain Med. 2003;4:321-30.

41. Hadley GR, Gayle JA, Ripoll J, et al. Post-herpetic neuralgia: a review. Curr Pain Headache Rep. 2016;20:17.

42. Lussier D, Beaulieu P, eds. Adjuvant Analgesics. New York: Oxford University Press; 2015.

43. U.S. Food and Drug Administration. FDA Adverse Events Reporting System (FAERS) public dashboard. Data as of November 29, 2018. Available at: http://prais.paho.org/en/launched-the-fda-adverse-event-reporting-system-faers-public-dashboard-2/. Accessed September 5, 2019.
44. U.S. Food and Drug Admininstration. FYs 2013-2017 GDUFA science and research report: transdermal drug products. Available at: https://www. fda.gov/ForIndustry/UserFees/GenericDrugUserFees/ucm605293.htm. April 4, 2018. Accessed September 5, 2019.

45. Banerjee S, Chattopadhyay P, Ghosh A, Datta P, Veer V. Aspect of adhesives in transdermal drug delivery systems. Int J Adhesion Adhesives. 2014;50:70-84.

46. Wokovich AM, Prodduturi S, Doub WH, Hussain AS, Buhse LF. Transdermal drug delivery system (TDDS) adhesion as a critical safety, efficacy and quality attribute. Eur J Pharm Biopharm. 2006;64(1):1-8. 